G392(P) INTRAVENOUS ANTIBIOTIC USE FOR SUSPECTED EARLYONSET BACTERIAL INFECTION IN HEALTHY NEONATES: A SINGLE-CENTRE CLOSED LOOP AUDIT

${ }^{1}$ ER Osen, ${ }^{2}$ GS Krishna. ${ }^{1}$ Ear Nose and Throat (ENT), Colchester Hospital University NHS Foundation Trust, Colchester, UK; '2Department of Paediatrics, Addenbrooke's Hospital, Cambridge, UK

\subsection{6/archdischild-2018-rcpch.381}

Aims We aimed to identify and reduce the number of healthy neonates started on intravenous antibiotics for suspected earlyonset bacterial infection in a district general hospital, and ensure that practice was commensurate with that set out in the relevant NICE (National Institute for Health and Care Excellence) guidance (CG149).

Methods A retrospective audit was performed using the clinical notes, drug charts and pathology reports of neonates who had been started on antibiotics in two 3 week periods, before and after intervention. The intervention was multifaceted and included the introduction of a neonatal sepsis pro forma and decision aid, $(\mathrm{P}) \mathrm{s}$ in relevant clinical areas, and teaching for junior and senior paediatricians.

Results The number of neonates commenced on antibiotics for suspected infection fell by $43 \%$ from 42 to 24 neonates, and compliance with absolute NICE criteria for commencement of antibiotics improved from $69 \%$ to $93 \%$. $76 \%$ of neonates in cycle 1 met NICE guidance for consideration of cessation of antibiotics at 36 hours but $77 \%$ of these neonates received more than 48 hours of antibiotics; similarly, in cycle 2, 73\% of neonates warranted consideration of cessation of antibiotics at 36 hours, but $91 \%$ of these received more than 48 hours of antibiotic treatment in total. In $77 \%$ of cases in cycle $1 \%$ and $82 \%$ of cases in cycle 2 , the documented rationale for continuing antibiotics past 36 hours in these healthy neonates was that blood culture results had not yet been reported.

Conclusion Our intervention was effective in reducing the number of healthy neonates started on intravenous antibiotics and improving compliance with NICE guidance for commencement of antibiotics. The proportion of neonates meriting a short duration of antibiotics was high despite improvement in following approved criteria, suggesting that there may be room for these criteria to be revised to capture fewer healthy neonates. However, our intervention was not successful in reducing duration of antibiotic use in healthy neonates. Establishing processes to accelerate pathology reporting could be the focus of further interventions to improve compliance with national guidelines and minimise unnecessary antibiotic exposure to healthy neonates.

\section{G393(P) JUST A 'SPOON FULL OF SUGAR' HELPS THE BREASTFEEDING RATES GO UP}

CP Darby. Neonatal, Southern Health and Social Care Trust, Craigavon, UK

\subsection{6/archdischild-2018-rcpch.382}

Aim A quality improvement initiative to improve the breastfeeding rates in the Trust to impact on the low breastfeeding rates in Northern ireland.

Method Northern Ireland currently has the lowest breastfeeding rates in the UK. Only $64 \%$ of mothers begin breastfeeding here, compared to $83 \%$ in England, $74 \%$ in Scotland and $71 \%$ in Wales. Mothers in Northern Ireland, who choose to breastfeed, also do so for a shorter period than breastfeeding mothers elsewhere in the UK. As a quality improvement (QI) strategy the areas of point of care that could be improved in order to advocate breastfeeding and optimise not only uptake but also the longevity of breastfeeding at point of discharge from hospital were analysed. The focus of the QI was to change the neonatal hypoglycaemia protocol to eliminate the use of milk formula as a substitute for breastmilk for infants with transient hypoglycaemia. Through critical analysis of the evidence the Trust guideline was replaced with a guideline that replace milk formula with $40 \%$ Glucose gel in combination with breastmilk/breastfeeding to improve neonatal hypoglycaemia management.

Results With the removal of milk formula as the option to treat neonatal hypoglycaemia, mothers were not made to feel inadequate when trying to establish breastfeeding. The use of the $40 \%$ glucose gel and its' ease of use and administration has been well received by midwives, doctors and parents. Breastfeeding rates have increased at the point of discharge from hospital. There has been significant savings within the Trust due to the reduction in milk formula consumption. Parents reported feelings of being 'supported' and 'empowered' when commencing and continuing breastfeeding. A reduction in neonatal admission for neonatal hypoglycaemia was apparent as a secondary outcome due to the change in guideline and intervention.

Conclusion Glucose gel use in combination with breastfeeding improves the support provided to breastfeeding mothers, leading to an increase of breastfeeding rates at the point of discharge from hospital. The successful quality improvement intervention has seen an improvement across two health directorates, Women and Child Health and Paediatrics. There was a reduction in neonatal admissions for neonatal hypoglycemia with improvement outcomes for infants as breastfeeding rates improve.

\section{G394(P) LEARNING FROM PICU TRANSFERS FROM A PAEDIATRIC EMERGENCY DEPARTMENT AND PAEDIATRIC WARD}

S Cuevas-Asturias, S Grossman, T Maggs, J Runnacles, L Sinitsky. Paediatrics and Child Health, Royal Free London NHS Foundation Trust, London, UK

\subsection{6/archdischild-2018-rcpch.383}

Aims Local hospital Paediatricians often rely on updates from retrieval services for information about their patients transferred to Paediatric intensive care units (PICU). This quality improvement project aimed to increase local hospital knowledge about their PICU transfers and identify areas for improvement to enhance patient safety and clinical care.

Methods In November 2016 a new incident log was implemented to collect data on PICU transfers from the Emergency Department and Paediatric ward of one district general hospital. Between January and September 2017, a multi-disciplinary team met monthly to review the Electronic Document and Records Management (EDRM) system of all patients transferred to PICU in the previous month(s). The multidisciplinary team included medical and nursing representation from Paediatric, Emergency and Anaesthetic departments and the outreach resuscitation team. EDRM was reviewed using an adapted RECALL (Rapid Evaluation Cardiorespiratory Arrest with Lessons for Learning) tool. The RECALL tool provided a structured template for retrospective case note review of 